\title{
Feedforward correction of mirror misalignment fluctuations for the GEO 600 gravitational wave detector
}

\author{
J R Smith ${ }^{1}$, H Grote ${ }^{1}$, M Hewitson ${ }^{1}$, S Hild ${ }^{1}$, H Lück ${ }^{1}$, M Parsons ${ }^{2,3}$, \\ K A Strain ${ }^{2}$ and B Willke ${ }^{1}$ \\ ${ }^{1}$ Max-Planck-Institute for Gravitational Physics (Albert-Einstein-Institute) and \\ University of Hannover, Callinstr. 38, D-30167 Hannover, Germany \\ 2 Institute for Gravitational Research, Department of Physics and Astronomy, \\ University of Glasgow, Glasgow G12 8QQ, UK \\ ${ }^{3}$ Department of Zoology, University of Cambridge, Downing Street, Cambridge CB2 3EJ, UK \\ E-mail: joshua.smith@aei.mpg.de
}

Received 29 April 2005, in final form 7 June 2005

Published 6 July 2005

Online at stacks.iop.org/CQG/22/3093

\begin{abstract}
The core instrument of the GEO 600 gravitational wave detector is a Michelson interferometer with folded arms. The five main optics that form this interferometer are suspended in vacuum by triple pendulums with quasimonolithic lower stages of fused silica. After installation of these pendulums in early 2003, a larger than expected coupling of longitudinal ground motion to tilt misalignment of the suspended optics was observed. Because of this, the uncontrolled misalignment of the optics during average conditions was several $\mu \mathrm{rad} \mathrm{Hz}{ }^{-1 / 2}$ in the frequency band around the pendulum resonance frequencies $(0.5-4 \mathrm{~Hz})$. In addition, it was found that longitudinal control signals applied to the intermediate pendulum stages also resulted in excessive mirror tilt. The resulting misalignment exceeded the level tolerable for stable operation of GEO 600. In order to reduce the level of mirror tilt, a bipartite feedforward system was implemented. One part feeds signals derived from seismic measurements to piezo-electric crystals in the stacks supporting the suspensions, reducing the longitudinal motion of the uppermost suspension points. The other applies tilt correction signals, derived from longitudinal control signals, to the intermediate level of the suspensions. The seismic feedforward correction reduces the root-mean-squared tilt misalignment of each main optic between 0.1 and $5 \mathrm{~Hz}$ by about $10 \mathrm{~dB}$, typically. The intermediatemass feedforward correction reduces the differential tilt misalignment of the Michelson interferometer by about $10 \mathrm{~dB}$ between 0.1 and $0.8 \mathrm{~Hz}$, typically.
\end{abstract}

PACS numbers: $04.80 . \mathrm{Nn}$, 95.55.Ym, 95.75.Kk, 02.30.Yy

(Some figures in this article are in colour only in the electronic version) 


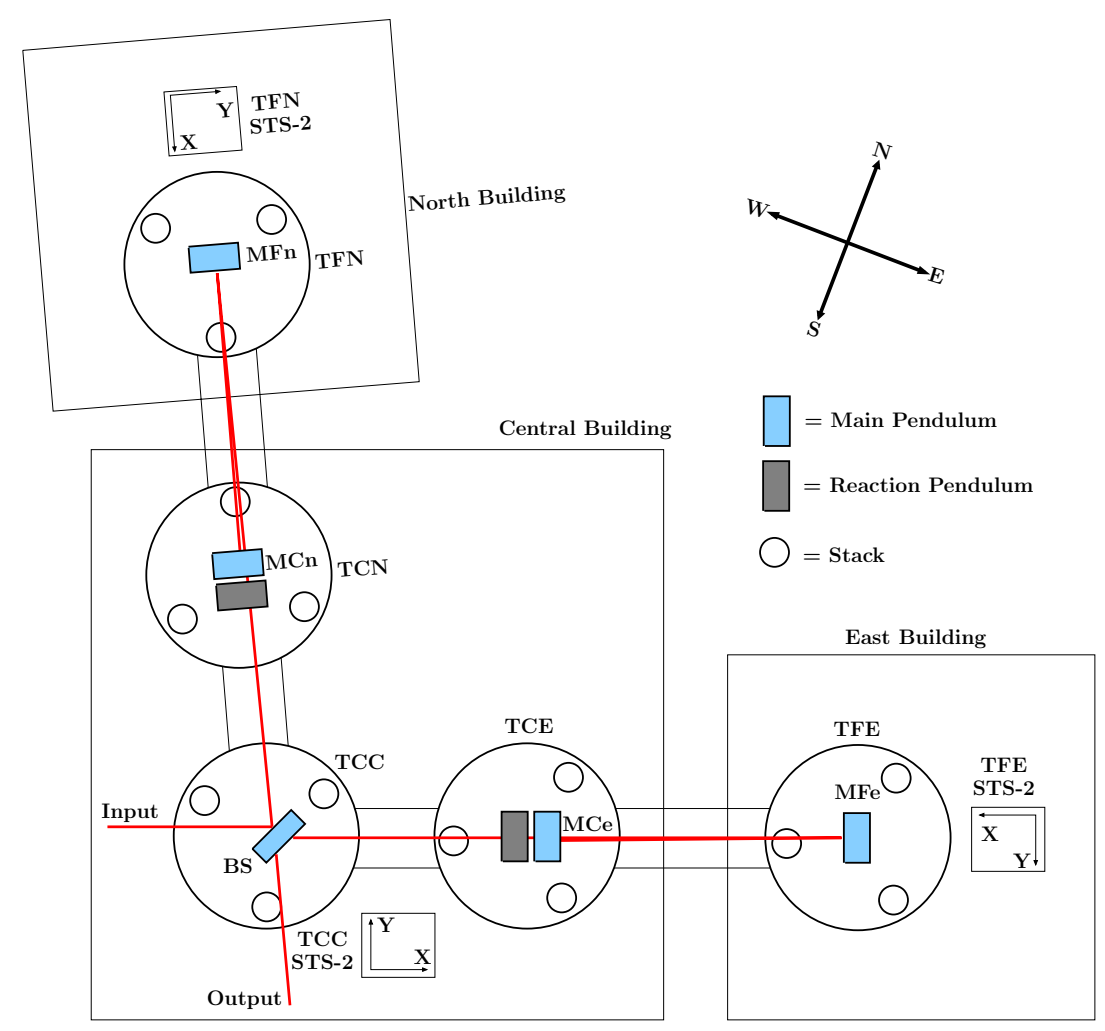

Figure 1. Simplified layout of the GEO 600 Michelson interferometer showing the position of the main vacuum tanks and suspensions and the position and orientation of the seismometers (denoted STS-2, see section 4). The tanks housing the near mirrors (TCE and TCN) are separated from TCC by roughly $1 \mathrm{~m}$, and the end buildings are separated from TCC by about $600 \mathrm{~m}$. The arms form an angle of $94^{\circ}$, and are folded once each, in the following way. The near mirrors MCe and MCn are displaced vertically from the other mirrors such that the beams from the BS first pass under the near mirrors, then are directed to the near mirrors by the far mirrors.

\section{Introduction}

GEO 600 is a long-baseline dual-recycled Michelson interferometer [1] that is part of an international network of interferometric gravitational wave detectors [2-4] designed to detect gravitational waves from astrophysical events. For more information about the design and performance of GEO 600 , see $[5,6]$.

The core instrument of GEO 600 is a Michelson interferometer (MI), with two nearly orthogonal folded arms, each with a geometric length of $600 \mathrm{~m}$. A simplified ${ }^{4}$ layout of the MI is shown in figure 1. The beamsplitter, BS, near mirrors, MCe and MCn, and far mirrors, MFe and MFn, are suspended in high vacuum as triple pendulums [7] in order to reduce the coupling of seismic and other noises into the detector output in the frequency band over which GEO 600 is designed to be sensitive to gravitational wave events $(50-6000 \mathrm{~Hz})$. Each of these pendulums has a quasi-monolithic fused silica lower stage $[8,9]$ in order to reduce the level of thermal noise that couples into the detector output.

4 The power- and signal-recycling mirrors, which, together with the MI, create the dual-recycled MI [1], have been omitted from this figure, and the following discussion, for simplicity. 
After the installation of these triple-pendulum mirror suspensions in 2003, several problems were encountered that led to difficulties for the operation of GEO 600. A larger than expected mismatch in the radii of curvature of the end mirrors was measured, and found to be detrimental to the locking and stability of the interferometer. To compensate for this, a ring-heater was installed behind MFe [10]. In addition, the main optics of the MI were found to exhibit more misalignment around their pendulum frequencies $(0.5-4 \mathrm{~Hz})$ than they had shown with steel wire test triple-pendulum suspensions. The reasons for this are discussed further in section 3 . The resulting misalignment was found to reduce the acquisition speed and stability of the interferometer lock, especially during periods of above-average seismic noise. A similar problem was encountered and solved by the TAMA group; for their reports, see [11, 12].

We have developed a bipartite system, using feedforward, in order to reduce the misalignment fluctuations of the core optics of GEO 600. The following section provides a calculation of the noise reduction of a feedforward system, as a function of the error of the correction signal with respect to the disturbance, as motivation for sections 4 and 5 in which the design and operational performance of the two parts of the system are discussed. Throughout this paper we will refer to motion along the axis perpendicular to the optical coating surface of an optic as longitudinal motion. Misalignment of an optic that produces vertical or horizontal misalignment of the reflected beam will be referred to as tilt or rotation, respectively.

\section{Feedforward theory}

Feedforward is a noise cancelling technique that works by sensing a disturbance before it effects a system, and reducing the disturbance's effect on the system predictively by applying a correction signal that is the measured disturbance multiplied by the known transfer function coupling the disturbance to the system. Although less versatile than feedback, feedforward has the advantage of being unconditionally stable, when acting alone. When combined with feedback, feedforward can reduce the required range of the feedback loop and/or improve its stability. The use of feedforward for the work described in this paper was driven by a lack of appropriate sensors for feedback control for the system discussed in section 4 and by simplicity of implementation for that discussed in section 5. Feedforward has already been demonstrated as a successful technique for reducing seismic noise coupling into long-baseline interferometers; see, e.g. [13].

The error of a feedforward system can be expressed as the frequency-dependent amplitude that remains after the subtraction of the correction signal from the disturbance in the system. For a disturbance of amplitude $\mathrm{A}_{\mathrm{d}}(f)$ and a correction signal of amplitude $\mathrm{A}_{\mathrm{c}}(f)$, this error is

$\epsilon\left(\mathrm{A}_{\mathrm{d}}(f), \mathrm{A}_{\mathrm{c}}(f), \phi(f)\right)=\sqrt{\mathrm{A}_{\mathrm{d}}^{2}(f)+\mathrm{A}_{\mathrm{c}}^{2}(f)-2 \mathrm{~A}_{\mathrm{d}}(f) \mathrm{A}_{\mathrm{c}}(f) \cos (\phi(f))}$,

where $\phi(f)$ is the phase difference between the two signals.

We can divide both sides of equation (1) by the disturbance amplitude $\mathrm{A}_{\mathrm{d}}(f)$ to produce a general frequency-dependent normalized error

$$
\hat{\epsilon}\left(\frac{\mathrm{A}_{\mathrm{c}}}{\mathrm{A}_{\mathrm{d}}}(f), \phi(f)\right)=\sqrt{\left(\frac{\mathrm{A}_{\mathrm{c}}}{\mathrm{A}_{\mathrm{d}}}(f)\right)^{2}-2 \frac{\mathrm{A}_{\mathrm{c}}}{\mathrm{A}_{\mathrm{d}}}(f) \cos (\phi(f))+1},
$$

which is a function of only the phase difference $\phi(f)$ and the amplitude ratio $\mathrm{A}_{\mathrm{c}}(f) / \mathrm{A}_{\mathrm{d}}(f)$. Surface and contour plots of this function are shown in figure 2. For a feedforward system to suppress a disturbance, e.g. by a factor of 10, the amplitude ratio and phase difference must be accurate enough to fall within the 0.1 contour space, i.e. within about $10 \%$ in amplitude and $5^{\circ}$ in phase. 

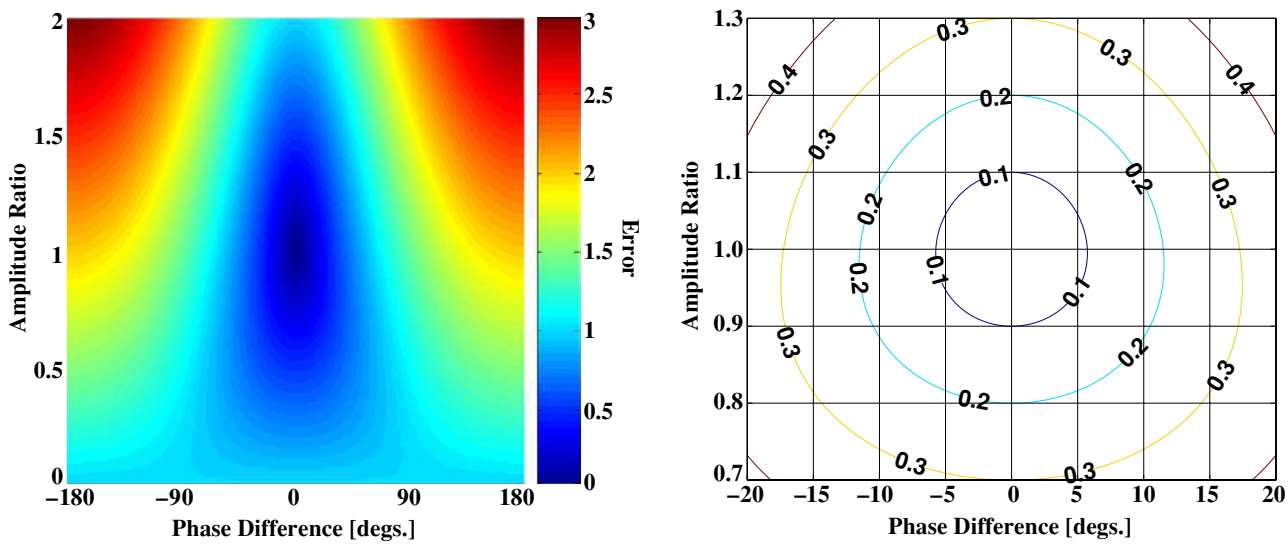

Figure 2. Feedforward error versus amplitude ratio and phase difference of the disturbance and correction signals.

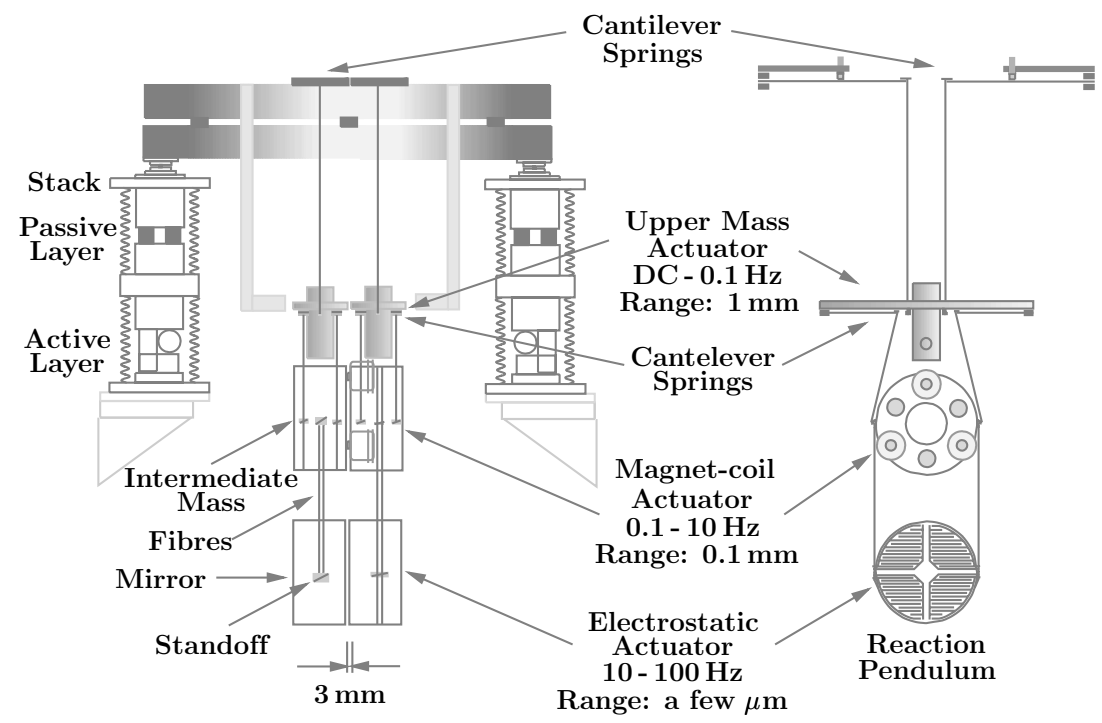

Figure 3. Diagram of a GEO 600 quasi-monolithic triple-pendulum suspension and reaction pendulum. Also shown are the ranges and frequency bands for each of the three hierarchical actuators used to control the longitudinal degree of freedom of the MI.

\section{Enhanced longitudinal to tilt coupling}

\subsection{Mechanism for enhanced coupling}

A diagram of a GEO 600 quasi-monolithic triple-pendulum suspension is shown in figure 3. The upper mass is suspended via two stainless steel wires from a pair of maraging (precipitation hardened) steel cantilever spring blades [7] that are mounted to a hexagonal rotational stage. Four steel cantilever blades are mounted to the bottom of the upper mass, and from these, the fused silica intermediate mass (IM) is suspended via two stainless steel wire slings. The mirror (in the following we also refer to the $\mathbf{B S}$ as a mirror) is suspended from the IM via four 

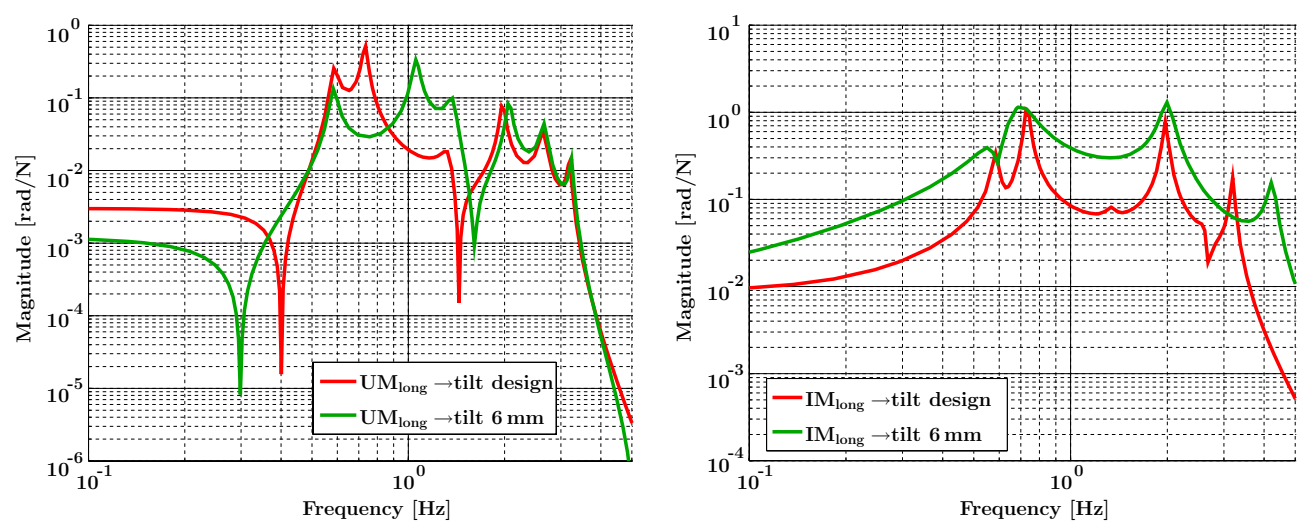

Figure 4. Modelled transfer functions for a GEO 600 triple-pendulum suspension from longitudinal force applied to the upper (left) and intermediate (right) mass, to tilt of the mirror. The red traces were modelled with fibre bending points at the designed heights, while the green traces are for fibre bending-point heights displaced from the design values by about $6 \mathrm{~mm}$.

tapered fused silica fibres [9] which are flame welded at each end to fused silica standoffs that are silicate bonded [14-16] to the sides of the two masses, thus forming a quasi-monolithic stage.

Experiments in Glasgow and Hannover prior to installation of the monolithic suspensions showed that attaching fibres with radii smaller than $150 \mu \mathrm{m}$, and constant over the entire fibre length, directly to the welding knobs of the fused silica standoffs, although desirable for giving high mechanical impedance and quality factor [17, 18], was not feasible with the current flame welding techniques. Therefore, the fused silica fibres used to suspend the optics were created with tapered ends. Each fibre has a radius of about $115 \mu \mathrm{m}$. This tapers to roughly $3 \mathrm{~mm}$ over a length of about $7 \mathrm{~mm}$, resulting in a 'neck' shape at both ends. The effect of these necks on the bending points of the fibres was not taken fully into account for the placement of the standoffs, and thus the height of the fibre bending points for pendulum-mode frequencies is about $7 \mathrm{~mm}$ above the height of the mirror centre of mass (COM) and the same amount below the COM of the IM. The design for the GEO 600 triple pendulums was for all wires and fibres to have bending points $1 \mathrm{~mm}$ away from (above or below depending on the direction of increased static stability) the COM of each of the three masses of the triple pendulum [7], thus the fibre bending-point heights are $6 \mathrm{~mm}$ away from their design values.

In order to estimate the effects of the non-optimal fibre bending points on the coupling of the various degrees of freedom of the triple pendulum, a simplified model was developed using Simulink in Matlab. Figure 4 shows modelled transfer functions from longitudinal force applied to the upper and intermediate pendulum stages, to mirror tilt for the design bending-point heights and for those displaced by $6 \mathrm{~mm}$ from the design values. According to the model, the displaced bending heights have an adverse effect on the transfer functions to mirror tilt. Comparing the model with offset bending points to that with the design values shows that, for the former, longitudinal forces applied to the upper mass result in about a factor of 10 more tilt at $1 \mathrm{~Hz}$ (although less at $0.7 \mathrm{~Hz}$ ). In addition, longitudinal forces applied to the intermediate mass will result in more tilt at nearly all pendulum-mode frequencies. It is also possible that other parameters of the suspensions may have changed during reinstallation, contributing to the observed higher level of tilt, however the offset bending points appear to have been the primary reason for this. 


\subsection{Effects of enhanced coupling}

After installation of the monolithic suspensions, when the interferometer was unlocked and the automatic beam-alignment (auto-alignment) system [20] was off, misalignment of each of the five MI optics during normal seismic conditions was a few $\mu$ rad root-mean-squared (RMS), dominated by tilt at pendulum resonance frequencies. Simulations done with FINESSE [19], have shown that this level of misalignment is enough to reduce the quality of the error signals used during lock acquisition [1] noticeably, sometimes making them invalid, and is thus detrimental to the lock acquisition process ${ }^{5}$. Longitudinal seismic motion, local to each suspension, was measured to be the dominant cause of this mirror tilt in the unlocked state.

Once in lock, the longitudinal degree of freedom of the MI is controlled by a servo to hold it at a dark fringe [1]. The range required to keep the MI at its operating point for long periods of time is quite large $(\approx 1 \mathrm{~mm})$ and thus the longitudinal feedback is applied via split paths to each of the three pendulum stages hierarchically, such that the largest signals are applied at the top of the suspension to benefit most from the filtering of the pendulum stages at higher frequencies. Longitudinal control signals are applied from dc to $0.1 \mathrm{~Hz}$ at the magnet-coil upper mass drives of MFe and MFn, from 0.1 to $10 \mathrm{~Hz}$ at the magnet-coil intermediate-mass drives of MCe and MCn, and above $10 \mathrm{~Hz}$ by electrostatic drives at the mirror level of MCe and MCn (see figure 3).

Because the arms of the GEO $600 \mathrm{MI}$ are folded, longitudinal motion of the far mirrors, MFe and MFn, has twice the influence of that of the near mirrors on the MI error signal. Thus, assuming each mirror to move roughly the same amount, the longitudinal correction signals that are applied to the near mirrors above $0.1 \mathrm{~Hz}$ are at least twice as large as the longitudinal motions of these mirrors. Tilt introduced by the resulting forces applied to the intermediate masses of MCe and MCn, and enhanced by the coupling described above, is the dominant cause of misalignment between 0.2 and $2 \mathrm{~Hz}$ in the locked state. Although the differential alignment of the MI is controlled during normal operation by the auto-alignment system, the residual RMS tilt misalignment without feedforward and under normal seismic conditions is still about $10 \mathrm{nrad}$ (dominated by resonances at approximately $0.6,1.2$ and $2.2 \mathrm{~Hz}$ ). This level of misalignment reduces the length of locked stretches, and is too large for stable operation of GEO 600 in its final configuration.

\section{Seismic feedforward correction}

The active layers of the seismic isolation stacks were designed for use as actuators in a feedforward system to reduce the relative motion of the mirrors at the microseismic peak at around $0.2 \mathrm{~Hz}$ [7]. During the commissioning of GEO 600 it became apparent that lock acquisition of the MI [1] could be made to work, under normal seismic conditions, with no active seismic isolation. Once in lock, longitudinal global control applied to the intermediate masses of the near mirrors had sufficient range $(0.1 \mathrm{~mm})$ and loop gain to reduce the longitudinal motions associated with the microseismic peak to a level that did not contribute significantly to the RMS deviation of the MI from its operating point. However, after installation of the final suspensions, large mirror misalignment, resulting from the enhanced coupling of longitudinal motion of the uppermost suspension point to mirror tilt discussed in the previous section, necessitated that an active seismic isolation system be used. A feedforward system was developed that reduces mirror tilt during the lock acquisition process, as well as 5 According to simulations, the tolerable level of misalignment during lock acquisition could be about one order of
magnitude smaller than this for the final (high finesse) configuration of GEO 600 . 

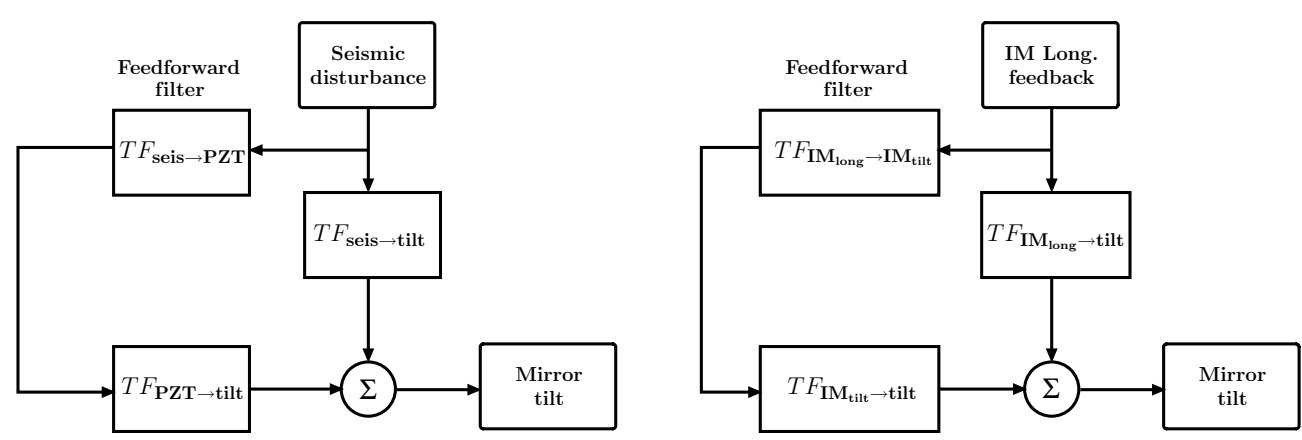

Figure 5. Block diagrams of the seismic (left) and intermediate-mass (right) feedforward systems.

the tilt signal input to the (currently gain-limited) MI auto-alignment system during lock. This section describes that system's design, implementation and operational performance.

\subsection{Design}

The goal of the seismic feedforward system is to reduce the longitudinal motion of the uppermost suspension point of each of the main optics as much as possible by measuring seismic motion in the longitudinal direction and feeding this, through appropriate filters, to the piezo-electric crystals (PZTs) in the active layers of the seismic isolation stacks. Seismic motion of the three foundations (one in each building), on which the tanks housing the core optics of the MI are built, is measured using three broadband tri-axial STS- 2 seismometers ${ }^{6}$. The approximate placement and orientation of these seismometers with respect to the MI is shown in figure 1 . In both end stations, the $x$-axis of the STS- 2 is aligned with the interferometer beam axis, and in the central building the $x$-axis is aligned with the beam travelling in the east arm (all to within a few degrees). Thus the longitudinal correction signal for the far mirrors and MCe require no adjustment of their reference frames. Because the arms of the MI are not exactly perpendicular (they form an angle of 94\%), the signals for the BS and MCn are defined by linear combinations of the $x$ - and $y$-axes of the STS-2 located in the central building.

\subsection{Implementation}

A block diagram of the seismic feedforward system is shown in figure 5. The filter required to transform the seismometer output signals into correction signals suitable for application to the PZTs, TF $_{\text {seis } \rightarrow \text { PZT }}$, was created by fitting the ratio of two measured transfer functions with a pole/zero model. The transfer functions from the seismometer signals to mirror tilt, $\mathrm{TF}_{\text {seis } \rightarrow \text { tilt }}$, and from a signal voltage applied to the PZTs to mirror tilt, $\mathrm{TF}_{\mathrm{PZT} \rightarrow \text { tilt }}$, were measured using optical levers (co-located HeNe laser and quadrant photodetectors set up to reflect off the mirror of interest). Division of these yielded the desired transfer function

$$
\mathrm{TF}_{\text {seis } \rightarrow \mathrm{PZT}}=\frac{\mathrm{TF}_{\text {seis } \rightarrow \text { tilt }}}{\mathrm{TF}_{\mathrm{PZT} \rightarrow \text { tilt }}} .
$$

The derived transfer function and the pole/zero filter used for MCe feedforward are shown in figure 6. The ratio of the transfer function measurements is valid from about $0.3 \mathrm{~Hz}$ (below which seismic motion dominated the motion we were able to produce using the PZTs) to about $3.5 \mathrm{~Hz}$ (above which the transfer function from longitudinal motion to mirror tilt falls

\footnotetext{
6 Streckeisen AG Messgeräte, Dättlikonerstrasse 5, CH-8422 Pfungen, Switzerland.
} 

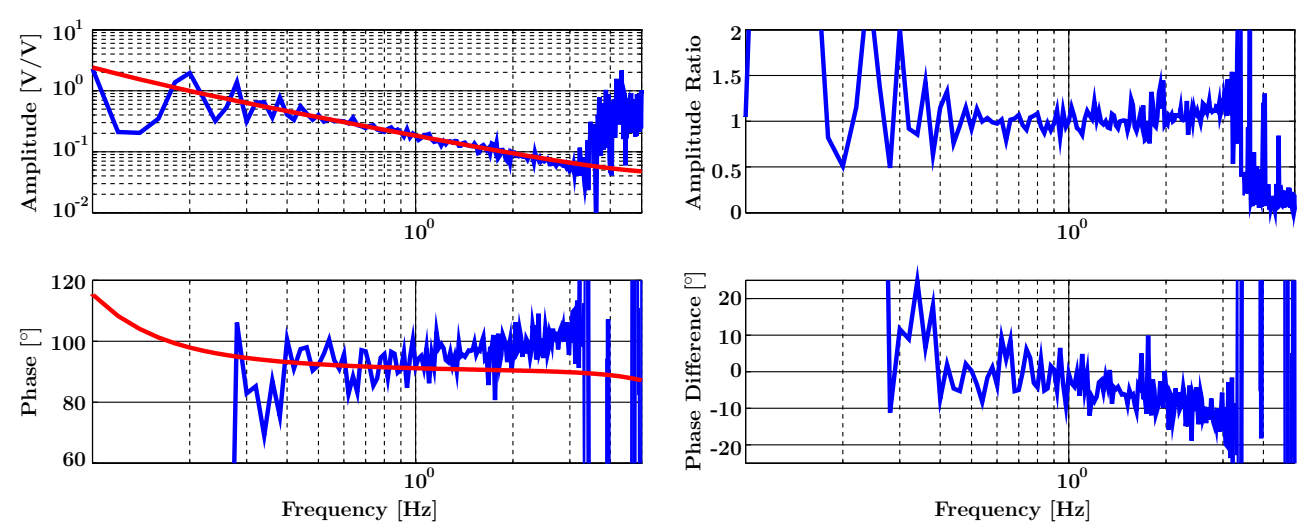

Figure 6. The derived transfer function $\mathrm{TF}_{\text {seis } \rightarrow \mathrm{PZT}}$ for MCe and the filter used to approximate it. The left plots show the magnitude and phase of the transfer function (blue) and filter (red), and the right plots show the amplitude ratio and phase difference.

off steeply and sensing noise from the quadrant photodetector limits). The filters were designed to have good amplitude and phase agreement over this frequency range. The magnitude ratio of, and phase differences between, $\mathrm{TF}_{\text {seis } \rightarrow \text { PZT }}$ and the designed filter are shown in figure 6 . The agreement is within $10 \%$ in amplitude and $5^{\circ}$ in phase over the range $0.6-1.2 \mathrm{~Hz}$.

In the central station the filters were implemented digitally using SIMULINK and dSPACE. In the end stations, where no digital systems were available at the time of implementation, the filters were created using analog electronics. No significant difference in the behaviour of the two systems was observed.

\subsection{Results}

Figure 7 shows the reduction of MCe tilt misalignment obtained using the seismic feedforward system during normal seismic conditions. Similar reduction was achieved for each core optic of the MI.

The first long-term operational test of this system was performed in the weeks prior to and during the coincident data-taking run S3 [6]. Two major problems were encountered. The first has to do with the fact that the STS-2 seismometers use three internal null-servoed sensors to measure seismic noise along three perpendicular axes and are thus sensitive to imbalances caused by tilt of the foundation on which they rest. Because of this, large signals (that cannot be distinguished from motion along one of the axes) are generated when people are working in one of the buildings, or when a vehicle passes nearby, etc. These signals, as inputs to the seismic feedforward, often result in enhanced low-frequency mirror motion and saturation of the control signals. The second is that the microseismic peak, which often varies by more than an order of magnitude on daily timescales at the GEO 600 site, sometimes creates motions that exceed the range of the $\mathrm{PZTs}^{7}$. During times when microseismic motions are large, the control signals saturate much of the time, greatly reducing the performance of the system. This problem could, in principle, be alleviated by an adaptive filtering scheme around the microseismic peak. This would, however, require installation of digital control systems in the two end buildings of GEO 600.

7 The rated range of the PZTs is sufficient to cancel the microseismic disturbances. However, several PZTs were damaged when they were driven with up to $230 \mathrm{~V}$, the rated voltage limit. To avoid further damage, the applied voltage was limited to $50 \mathrm{~V}$. 


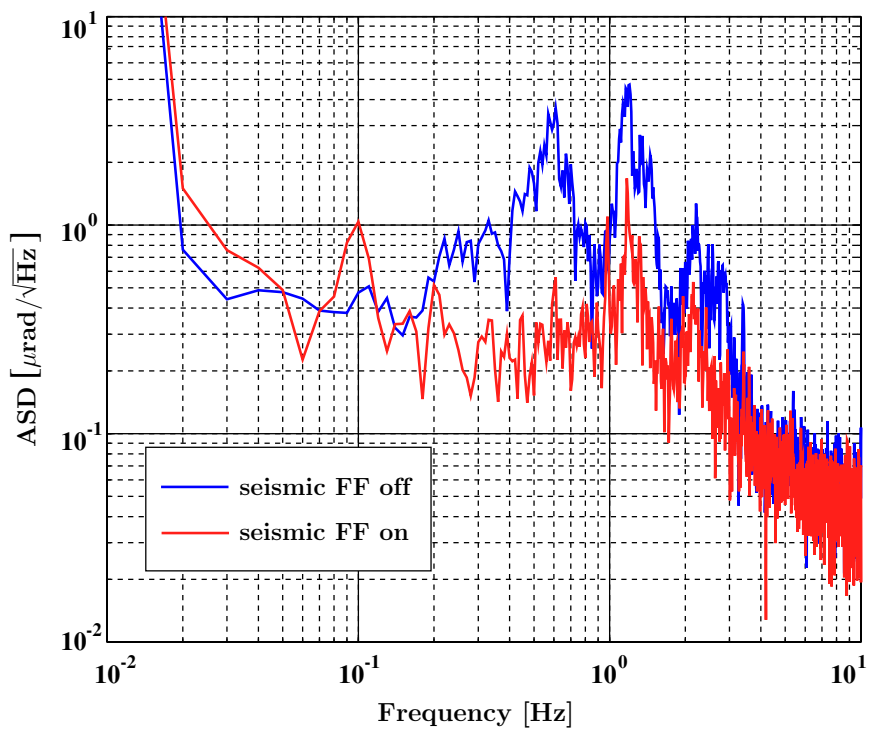

Figure 7. Reduction of MCe tilt achieved when using the seismic feedforward system.

\section{Intermediate-mass feedforward correction}

As discussed in section 3, during interferometer operation the longitudinal degree of freedom of the MI is locked to the dark fringe via longitudinal control signals applied to the upper masses of the far suspensions, and the intermediate and mirror masses of the near suspensions. The forces applied to the near mirror intermediate masses cause misalignment of the near mirrors via the inherent coupling of the various degrees of freedom of the suspensions and the fact that the intermediate-mass drives are not perfectly orthogonalized. This misalignment is the dominant cause of MI tilt between 0.1 and $0.8 \mathrm{~Hz}$ in the locked state. Since the MI automatic alignment system, which is responsible for maintaining the differential alignment of the MI, is gain limited at these frequencies, and since tilt misalignment fluctuations are the dominant cause of the RMS misalignment of the MI, it is desirable to suppress the tilt misalignment that is input to the auto-alignment system around these frequencies.

\subsection{Design}

The goal of the intermediate-mass feedforward system is to reduce, as much as possible, the coupling of longitudinal feedback signals applied to the IMs to tilt of MCe and MCn. This is done by applying correction signals, derived from the longitudinal feedback, to the tilt inputs of the intermediate-mass drives. This system will likely be extended to provide a full orthogonalization the IM drives, as need arises.

\subsection{Implementation}

A block diagram of the intermediate-mass feedforward system is shown in figure 5. The filter required to prepare tilt correction signals for the IM drive from longitudinal feedback signals, $\mathrm{TF}_{\text {IMlong } \rightarrow \text { IMtilt }}$, was derived by fitting the ratio of two transfer functions to a pole/zero model. For each near mirror, the transfer function from signals applied to the longitudinal inputs of 

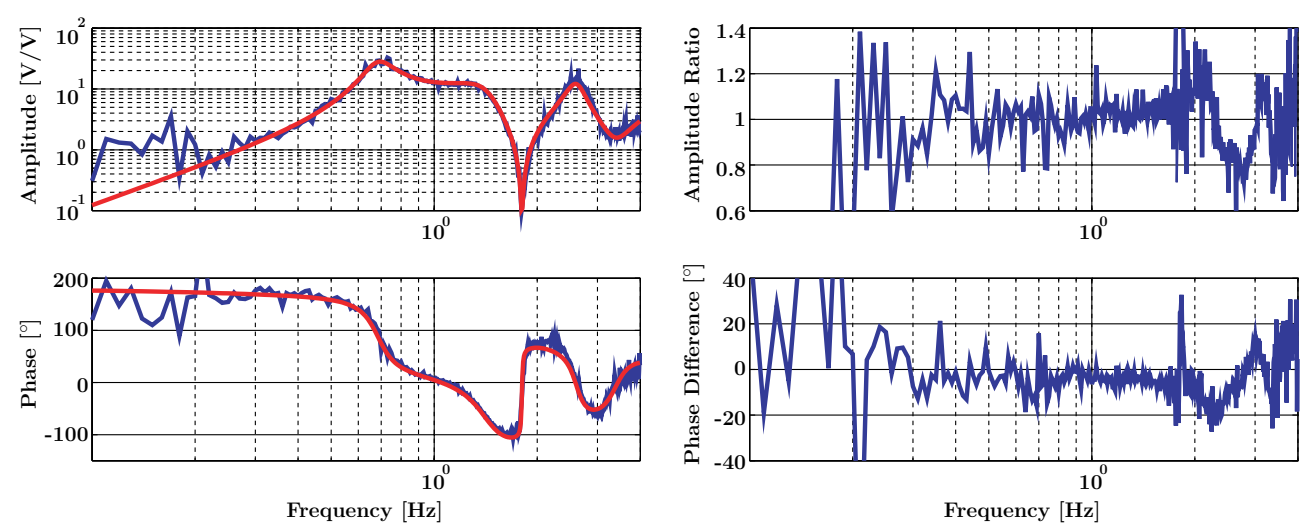

Figure 8. The derived filter $\mathrm{TF}_{\mathrm{IM}} \mathrm{Tlong} \rightarrow \mathrm{IM}$ tilt for MCe and the filter used to approximate it. The left-hand plots show the magnitude and phase of the measured (blue), and pole/zero modelled (red), filters. The right-hand plots show the amplitude ratio and phase difference of these.

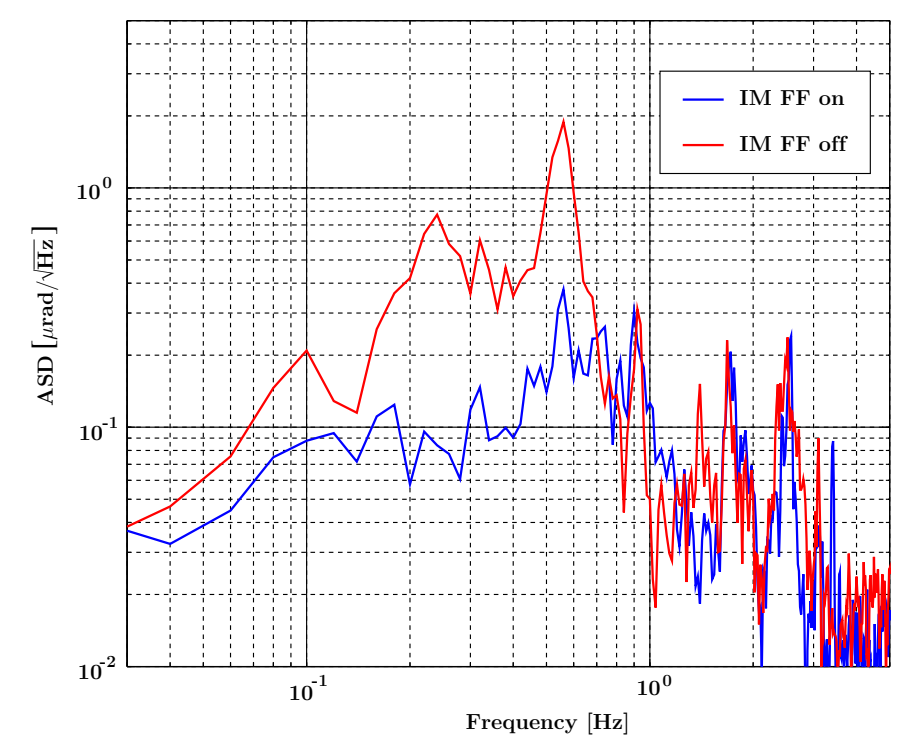

Figure 9. Reduction of the MI fast auto-alignment tilt feedback signal given by the intermediatemass feedforward system. The seismic feedforward system was operating for both measurements.

the IM drive, to mirror tilt, $\mathrm{TF}_{\mathrm{IMlong} \rightarrow \text { tilt }}$, and from signals applied to the tilt inputs of the IM drives, to mirror tilt, $\mathrm{TF}_{\mathrm{IM} \text { tilt } \rightarrow \text { tilt }}$, were measured using optical levers (as described in section 4). Division of these yielded

$$
\mathrm{TF}_{\text {IMlong } \rightarrow \text { IMtilt }}=\frac{\mathrm{TF}_{\text {IMlong } \rightarrow \text { tilt }}}{\mathrm{TF}_{\text {IMtilt } \rightarrow \text { tilt }}} .
$$

The derived transfer function for MCe and the pole/zero filter fit to it are shown in figure 8, along with the amplitude ratio and phase difference of the measured transfer function and fit filter. For MCe, the amplitude ratio and phase difference are within $10 \%$ and $10^{\circ}$ over the range $0.2-2 \mathrm{~Hz}$, while for $\mathbf{M C n}$, the agreement was slightly worse. The filters for both MCe and MCn were implemented digitally using SIMULINK and dSPACE. 


\subsection{Results}

Figure 9 shows the reduction of the MI fast auto-alignment tilt feedback signal given by the IM feedforward tilt correction system for MCe and MCn. In addition to this, a slight reduction of the rotation auto-alignment feedback was also observed. The control signals for the MI length control were not noticeably effected. This system was used, and operated stably, during the entire S3 science run [6].

\section{Summary}

After installation of the final triple-pendulum suspensions for the Michelson interferometer core optics, a larger than expected level of tilt misalignment of the suspended optics existed, due to non-optimal bending points of the suspension fibres. We developed a two-part system, using feedforward, to reduce this tilt misalignment. The seismic feedforward system reduces the uncontrolled misalignment of each MI optic between 0.1 and $5 \mathrm{~Hz}$ by about $10 \mathrm{~dB}$, typically. The reliability of this system is, however, diminished by the limited range of the PZTs used, and by the sensitivity of the STS- 2 seismometers to imbalances. The intermediatemass feedforward system reduces tilt misalignment of the Michelson interferometer by about $10 \mathrm{~dB}$ between 0.1 and $0.8 \mathrm{~Hz}$, typically. This system will be extended in the future to provide a complete orthogonalization of the IM drive. The reduction in tilt misalignment fluctuations given by the complete system should result in faster lock acquisition and longer lock stretches, and a reduction of the influence on the detector output of GEO 600, of any noise sources with couplings that depend on the residual misalignment of the Michelson interferometer.

\section{Acknowledgments}

The authors thank Joe Giaime from LIGO for help and advice pertaining to the seismic feedforward system. We thank Stefan Goßler for useful discussions concerning the suspension systems and providing figure 3. We also thank LIGO, and especially Fred Raab, for loaning us three STS-2 seismometers. We are grateful for support from PPARC and the University of Glasgow in the UK, and the BMBF and the state of Lower Saxony in Germany.

\section{References}

[1] Grote H, Freise A, Malec M, Heinzel G, Willke B, Lück H, Strain K A, Hough J and Danzmann K 2004 Dual recycling for GEO 600 Class. Quantum Grav. 21 S473

[2] Takahashi R et al 2004 Status of TAMA300 Class. Quantum Grav. 21 S403

[3] Frasconi F et al 2004 Status of VIRGO Class. Quantum Grav. 21 S385

[4] Sigg D et al 2004 Commissioning of LIGO detectors Class. Quantum Grav. 21 S409

[5] Willke B et al 2002 The GEO 600 gravitational wave detector Class. Quantum Grav. 191377

[6] Smith J R et al 2004 Commissioning, characterization and operation of the dual-recycled GEO 600 Class. Quantum Grav. 21 S1737

[7] Plissi M V, Torrie C I, Housman M E, Robertson N A, Strain K A, Ward H, Lück H and Hough J 2000 GEO 600 triple pendulum suspension system: seismic isolation and control Rev. Sci. Instrum. 712359

[8] Smith J R, Cagnoli G, Crooks D R M, Fejer M M, Goßler S, Lück H, Rowan S, Hough J and Danzmann K 2004 Mechanical quality factor measurements of monolithically suspended fused silica test masses of the GEO 600 gravitational wave detector Class. Quantum Grav. 21 S1091

[9] Goßler S, Cagnoli G, Crooks D R M, Lück H, Rowan S, Smith J R, Strain K A, Hough J and Danzmann K 2004 Damping and tuning of the fibre violin modes in monolithic silica suspensions Class. Quantum Grav. $21 \mathrm{~S} 923$

[10] Lück H, Freise A, Goßler S, Hild S, Kawabe K and Danzmann K 2004 Thermal correction of the radii of curvature of mirrors for GEO 600 Class. Quantum Grav. 21 S985 
[11] Takamori A et al 2002 Mirror suspension system for the TAMA SAS Class. Quantum Grav. 191615

[12] Ando $\mathrm{M}$ et al 2001 Stable operation of a 300-m laser interferometer with sufficient sensitivity to detect gravitational-wave events within our galaxy Phys. Rev. Lett. 863950

[13] Giaime J A, Daw E J, Weitz M, Adhikari R, Fritschel P, Abbott R, Bork R and Heefner J 2003 Feedforward reduction of the microseism disturbance in a long-base-line interferometric gravitational-wave detector Rev. Sci. Instrum. $\mathbf{7 4} 218$

[14] Gwo D H 2001 Ultra precision and reliable bonding method US Patent 6284085 B1

[15] Sneddon P H, Bull S, Cagnoli G, Crooks D R M, Elliffe E J, Faller J E, Fejer M M, Hough J and Rowan S 2003 The intrinsic mechanical loss factor of hydroxy-catalysis bonds for use in the mirror suspensions of gravitational wave detectors Class. Quantum Grav. 205025

[16] Smith J R, Harry G M, Betzwieser J C, Gretarsson A M, Guild D A, Kittelberger S E, Mortonson M J, Penn S D and Saulson P R 2003 Mechanical loss associated with silicate bonding of fused silica Class. Quantum Grav. 205039

[17] Willems P A and Thattai M T 1999 Increased thermal noise in nonuniform fiber suspensions Phys. Lett. A 253 16

[18] Willems P, Sannibale V, Weel J and Mitrofanov V 2002 Investigations of the dynamics and mechanical dissipation of a fused silica suspension Phys. Lett. A 29737

[19] Freise A 2004 Frequency domain interferometer simulation with higher-order spatial modes Class. Quantum Grav. 21 S1067 (see also http://www.rzg.mpg.de/ adf)

[20] Grote H et al 2004 Alignment control of GEO 600 Class. Quantum Grav. 21 S441 